\title{
A Summary of the Fifth Annual Virology Education HIV Microbiome Workshop
}

\author{
Scott Sherrill-Mix, ${ }^{1}$ Kaleigh Connors, ${ }^{2}$ Grace M. Aldrovandi, ${ }^{3}$ Jason M. Brenchley, ${ }^{4}$ Charles Boucher, ${ }^{5}$ \\ Frederic D. Bushman, ${ }^{1}$ Ronald G. Collman, ${ }^{6}$ Satya Dandekar, ${ }^{7}$ Nichole R. Klatt, ${ }^{8}$ Laurel A. Lagenaur, ${ }^{9}$ \\ Roger Paredes, ${ }^{10}$ Gilda Tachedjian, ${ }^{11}$ Jim A. Turpin, ${ }^{12}$ Alan L. Landay, ${ }^{13}$ and Mimi Ghosh ${ }^{2}$
}

\begin{abstract}
In October of 2019, researchers and community members from around the world met at the NIH for the fifth annual International Workshop on Microbiome in HIV. New research was presented on the role of the microbiome on chronic inflammation and vaccine design, interactions of genetics, environment, sexual practice and HIV infection with the microbiome and the development and clinical trials of microbiome-based therapeutic approaches intended to decrease the probability of HIV acquisition/transmission or ameliorate sequelae of HIV. The keynote address by Dr. Jacques Ravel focused on his work on the vaginal microbiome and efforts to improve the analysis and resolution of microbiome data.
\end{abstract}

Keywords: HIV/SIV, microbiome, pathogenesis, therapeutics, comorbidities, transmission

\section{Introduction}

$\mathbf{T}$ HIS YEAR MARKED the fifth meeting of the International Workshop on Microbiome in HIV held at the NIH. ${ }^{1-4}$ In the past year, appreciation for the interplay between the human microbiome and HIV infection has continued to grow. New studies presented here help clarify the role of the microbiome on chronic inflammation and vaccine design and reveal the interaction between the effects of genetics, environment, sexual practice, and HIV infection on the microbiome. Further, reports on the development and clinical trials of microbiome-based therapeutic approaches investigate the potential of microbiome alterations to decrease the probability of acquisition/transmission or ameliorate sequelae of HIV.

Essential to all studies is the continued development and standardization of best methods for data analysis and consistency across studies and approaches. The keynote address by Dr. Jacques Ravel focused on his work to improve the analysis and resolution of microbiome data.

\section{Keynote Address}

The microbiome of the vagina appears to have significant effects on the rate of HIV acquisition ${ }^{5-8}$ and efficacy of antiretroviral prevention and treatment. ${ }^{9,10}$ The bacterial communities of the vagina have been categorized into patterns of abundance called Community State Types (CST) with most CST dominated by species of Lactobacilli and a single CST containing diverse communities with little representation of Lactobacillus (often labeled "CST IV" from its initial identification). ${ }^{11,12}$ This diverse CST is associated with genital inflammation and persistent bacterial vaginosis. ${ }^{11,13}$ Dr. Jacques Ravel from the University of Maryland discussed his seminal article from 2011, in which he used 16S sequencing to divide the microbial communities of the vagina

\footnotetext{
${ }^{1}$ Department of Microbiology, Perelman School of Medicine, University of Pennsylvania, Philadelphia, Pennsylvania, USA.

${ }^{2}$ Department of Epidemiology, The George Washington University, Washington, District of Columbia, USA.

${ }^{3}$ Department of Pediatrics, University of California, Los Angeles, Los Angeles, California, USA.

${ }^{4}$ Laboratory of Viral Diseases, NIAID, NIH, Bethesda, Maryland, USA.

${ }^{5}$ Department of Virosciences, Erasmus Medical Center, Erasmus University Rotterdam, Rotterdam, the Netherlands.

${ }^{6}$ Department of Medicine, Perelman School of Medicine, University of Pennsylvania, Philadelphia, Pennsylvania, USA.

${ }^{7}$ Department of Medical Microbiology and Immunology, University of California, Davis, Davis, California, USA.

${ }^{8}$ Department of Medicine, University of Minnesota, Minneapolis, Minnesota, USA.

${ }^{9}$ Osel, Inc., Mountain View, California, USA.

${ }^{10}$ Institut de Recerca de la SIDA IrsiCaixa i Unitat VIH, Universitat Autònoma de Barcelona, Universitat de Vic, Vic, Spain.

${ }^{11}$ Burnet Institute, Melbourne, Australia.

${ }^{12}$ Divison of AIDS, NIAID, NIH, Bethesda, Maryland, USA.

${ }^{13}$ Division of Gerontology, Department of Internal Medicine, Rush University Medical Center, Chicago, Illinois, USA.
} 
into five main CST. ${ }^{11}$ The techniques used to group the abundances into CST in this and similar articles result in CST that can be difficult to compare between studies, sensitive to the addition or removal of samples and challenging to interpret. To address this issue, Dr. Ravel and his group have developed a tool for improved CST classification called VALENCIA (VAginaL community state typE Nearest CentroId clAssifier). This classifier has improved resolution of CST allowing the original five CST to be split into 13 hierarchical CST.

Current research into the vaginal microbiome frequently combines metagenomics, metatranscriptomics, and proteomics. To enable analysis of these datasets, the Ravel group collected over 400 urogenital bacteria genomes and 1,500 metagenomes to build a human vaginal nonredundant gene catalog called VIRGO ${ }^{14}$ This catalog contains 0.95 million nonredundant genes compiled from 318 unique bacterial species. To allow analysis of genes and proteins across bacterial species, these nonredundant genes are further clustered into vaginal orthologous groups. Comparison to vaginal metagenomes from North American, African, ${ }^{8}$ and Chinese ${ }^{15}$ women showed coverage of more than $95 \%$ vaginal bacterial gene content. Analyses of these metagenomes also revealed the presence of distinct strains within each bacterial species leading to the concept of a metagenomic subspecies as the sum of unique genes belonging to a given species shared within a group of samples. These metagenomic subspecies can then be clustered to derive metagenomic CST reflecting bacterial species, strains, and gene content.

Application of these techniques to a longitudinal study of 3,620 reproductive-age women ${ }^{16}$ revealed associations between metagenomic CST and odds of incident sexually transmitted infection (STI). The importance of subspecies level assignments was highlighted when women whose metagenomic CST were dominated with a particular metagenomic subspecies of Gardnerella vaginalis and showed increased risk of STIs while CST dominated by another G. vaginalis strain significantly decreased risk. Interestingly, these two inferred subspecies have since been independently suggested to be separate species. ${ }^{17}$

Finally, Dr. Ravel discussed work on the relationship between Chlamydia trachomatis infection and lactic acid produced by the vaginal microbiome. Previous studies have shown that lactic acid produced by some but not other Lactobacillus species appears to inhibit $C$. trachomatis infection. ${ }^{18,19}$ Vaginal microbiota produce lactic acid in two isomers, $\mathrm{D}(-)$ and $\mathrm{L}(+)$. Research in the Ravel laboratory revealed that Lactobacillus differ in the ratio of $\mathrm{D}(-)$ or $\mathrm{L}(+)$ lactic acid produced and that Lactobacillus species that produce $\mathrm{D}(-)$ lactic acid were associated with protection. ${ }^{20}$ Transcriptomic analysis revealed that rather than a direct impact of $\mathrm{D}(-)$ lactic acid on chlamydial infection, epigenetic modifications involving histone deacetylase-controlled pathways were responsible for modulating immune responses to the pathogen. ${ }^{20}$

\section{Transmission and Prevention}

Understanding the role of the vaginal microbiome and inflammation in HIV transmission is critical to preventing spread. Dr. Lindi Masson from the University of Capetown discussed the use of metaproteomics to evaluate the vaginal microenvironment and better understand how mucosal inflammation associates with HIV acquisition risk in young women. ${ }^{13,21,22}$ Using data from the WISH (Women's Initiative in Sexual Health) study, Dr. Masson's team found that bacterial abundances estimated by metaproteomics were largely congruent with those found by $16 \mathrm{~S}$ sequencing, however, microbial function determined by proteomics seemed to predict inflammation more accurately than microbial species abundance. Particularly, cell wall and membrane biological processes and components were underrepresented in microbes from women with inflammation, whereas stress response, energy metabolism, and cell division were overrepresented in microbes from women with inflammation. To validate these results in vitro, 80 Lactobacillus species were isolated from vaginal samples and assessed for metabolic products and inflammation induction. Strains isolated from women with dysbiosis were more inflammatory in vitro and similar proteomic associations with inflammation were observed.

With bacterial vaginosis associated with increased risk for acquiring $\mathrm{HIV}{ }^{7,8}$ effective treatment is critical. However, antibiotic treatment of bacterial vaginosis has low cure rates and results in frequent recurrence. ${ }^{23-25}$ Dr. Craig Cohen from the University of California San Francisco reported on trials of the live biotherapeutic LACTIN-V, a formulation of naturally occurring Lactobacillus crispatus strain CTV-05, ${ }^{26,27}$ to reduce recurrence of vaginal dysbiosis. Initial trials have shown increased Lactobacillus colonization after antibiotic and LACTIN-V treatment ${ }^{28}$ (NCT00635622), and a phase $2 \mathrm{~b}$ trial testing efficacy in the prevention of bacterial vaginosis recurrence was recently completed ${ }^{29}$ (NCT02766023). In addition, a phase 2 trial investigating the effects of LACTIN-V on genital inflammation is beginning to enroll women at high risk for HIV in South Africa.

Since there is a strong link between race and bacterial vaginosis, ${ }^{11,30}$ it seems likely that there may be a genetic predisposition to dysbiosis. Dr. Supriya Mehta from the University of Illinois at Chicago reported on a genome wide association study searching for a link between genetic polymorphisms and bacterial vaginosis in a cohort of 252 heterosexual females. Several polymorphisms showed associations with bacterial vaginosis, community diversity, and the abundances of several inflammation-associated taxa. Promisingly, pathway analysis of these polymorphisms showed an enrichment in genes annotated as related to the urinal and genital tract.

The success of the ASPIRE (A Study to Prevent Infection with a Ring for Extended Use) and Ring study phase III trials of vaginal rings containing antiretrovirals ${ }^{31,32}$ has driven research to provide improved vaginal ring delivery technology. However, concerns remain regarding adverse effects from inflammation or microbial dysbiosis. Dr. Marc Baum from Oakcrest Institute of Science presented data from a Phase 1 randomized, placebo-controlled clinical trial of podintravaginal rings (IVR) ${ }^{33}$ used to deliver antiretroviral drugs. This approach previously provided $100 \%$ protection in macaques challenged with SHIV. ${ }^{34}$ For this safety trial, healthy women used pod-IVR containing antiretroviral drugs for 7 days. ${ }^{35}$ A single vaginal swab was used in a multiomic approach to assess community composition (16S sequence), 
structure (imaging), and community function (metabolomics and metaproteomics). Antiretrovirals were detected in vaginal as well as rectal samples and no significant changes in vaginal microbiomes were observed. Pod-IVR appears to offer a promising delivery technology for the fields of HIV and women's health.

Other preliminary data in this category that were presented in poster format included the effects of menstrual cup usage on vaginal microbiome and STI in Kenyan school girls (Cups and Community Health [CaCHe] study; Dr. Supriya Mehta from the University of Illinois at Chicago); screening of vaginal bacteria metabolites for effects on HIV replication (Kaitlin Marquis from the University of Pennsylvania); a meta-analysis on the effects of topical penile microbicide (Dr. Venkateshwaran Sivaraj from Guys and St. Thomas NHS Foundation Trust); associations between the bacteriome and virome in the female reproductive tract (Ferralita Madere from the University of Rochester); the protective effects of lactic acid producing Lactobacilli against short-chain fatty acid-induced cervicovaginal inflammation ${ }^{36}$ (Dr. Gilda Tachedjian from the Burnet Institute) and interference with tenofovir-mediated HIV inhibition in women with bacterial vaginosis (Dr. Richard Hunte from the Albert Einstein College of Medicine).

\section{Pathogenesis}

The oral mucosal barrier faces continuous challenge from diverse microbes, antigens, and mechanical damage. In the face of these challenges, the immune system must balance between surveillance and inflammatory response. ${ }^{37}$ Dysbiosis of the microbiome of the oral mucosa can lead to periodontitis, resulting in systemic microbial translocation and tissue destruction. ${ }^{38}$ Dr. Niki Moutsopoulos from the NIDCR/NIH discussed the role of proinflammatory Th17 cells $^{39,40}$ in the maintenance of oral microbiome and periodontal health. ${ }^{41}$ Using a murine model of periodontitis, her group showed that in healthy mice, physiologic damage from mastication is a trigger for accumulation of Th17. ${ }^{42}$ However, during disease, Th17 expansion appears to be dependent on the microbiome since antibiotic treatment (particularly those affecting the gram-negative anaerobic bacteria) reduces Th17 accumulation. ${ }^{43}$ Small molecule inhibitors or genetic knockout of Th17 cells reduced inflammation and bone loss in a mouse model ${ }^{43}$ suggesting that Th17 cells may represent both a driver of and therapeutic target for periodontitis. In support of this theory, humans with genetic defects in Th17 differentiation have reduced risk of periodontitis although they do suffer from increased risk of other pathologies. $^{43,44}$

The microbiome also plays a critical role in the development and maintenance of host immune function. ${ }^{45,46}$ The interplay between resident microbiota and viral pathogens is being increasingly appreciated as an important factor in pathogenesis. ${ }^{47-49}$ Dr. Megan Baldridge from the Washington University School of Medicine, presented two examples of these interactions in the regulation of intestinal norovirus infection by microbiota using a murine model. She found that persistent strains of murine norovirus, MNoV, infect and maintain a reservoir in rare intestinal epithelial cells called tuft cells. ${ }^{50-52}$ The abundance of these tuft cells and replicative capacity of $\mathrm{MNoV}$ is greatly reduced by an- tibiotic treatment, ${ }^{52}$ but is restored by fecal transplant or supplementation of a single species Bacteroides ovatus. Why $B$. ovatus? It appears that $B$. ovatus produces a succinate metabolite that is known to stimulate tuft cell growth. ${ }^{53-55}$ When succinate production is knocked out, B. ovatus can no longer restore $\mathrm{MNoV}$ replication. However, if succinate is supplemented, MNoV replication is restored to antibioticsfree levels. In another novel interaction, Dr. Baldridge reported on an unknown factor that protects against $\mathrm{MNoV}$ infection in immunodeficient mice. Immunodeficient $\operatorname{Rag} 1^{-/-}$ Il $2 \mathrm{rg}^{-/-}$mice are resistant to $\mathrm{MNoV}$ infection while $\mathrm{Rag}^{-/-}$ are normally sensitive. However, $\operatorname{Rag} 1^{-/-}$mice cohoused with or receiving fecal transplants from $\mathrm{Rag} 1^{-/-} \mathrm{Il} 2 \mathrm{rg}^{-/-}$mice were also resistant. Interestingly, even $0.2 \mu \mathrm{m}$ filtered fecal transplants transferred resistance, but the effect was lost following heat, ultraviolet, or proteinase $\mathrm{K}$ treatment. Sequencing and further testing revealed that this factor was likely a murine astrovirus present only in immunocompromised mice and that this astrovirus appears to induce IFN- $\lambda$ leading to reduced MNoV replication. ${ }^{56}$

Chronic HIV/SIV infection has been linked to translocation of microbes from the gut and chronic inflammation. ${ }^{57-59}$ Microvesicles released by the gastrointestinal epithelium may contribute to antimicrobial defenses and immune regulation in the gut. ${ }^{60-63}$ To determine whether the antimicrobial cargo in intestinal microvesicles is dysfunctional in SIV infection, Dr. Alexandra Ortiz O'Sick from Dr. Jason Brenchley's group at the NIAID/NIH isolated fecal microvesicles from infected macaques. Characterization of the microRNA (miRNA) content of these microvesicles revealed potential changes in miRNAs and beta-defensin abundance in SIV-infected macaques. In addition, coculture of gut bacteria Lactobacillus salivarius, a bacteria commonly isolated in assays of translocation, with microvesicles from SIV+/SIV- animals revealed potential effects on bacterial growth.

Dr. Leila Giron from Dr. Mohamed Abdel-Mohsen's laboratory at the Wistar Institute discussed the glycomic determinants of gut microbial dysbiosis and translocation during suppressed HIV infection. An emerging model suggests that gut glycosylation is a critical homeostatic mediator. ${ }^{64}$ In a cohort of HIV suppressed individuals, glycomic profiles of colonic biopsies showed evidence of higher levels of sialidase-releasing microbes associated with gut hyposialylation and a dysbiotic pattern of lower microbial diversity. On the contrary, fucosylation appeared to be protective and associated with higher microbial diversity and lower dysbiosis. RNA-Seq analysis revealed a connection between gut hyposialylation and the activation of the inflammasome-mediator eIF2 signaling, whereas fucosylation was linked to the inhibition of this signaling. These data point to potential connections between gut glycomic patterns in HIV-mediated inflammation and bacterial translocation. ${ }^{65}$

\section{Comorbidities}

People living with HIV (PLHIV) often suffer from metabolic derangements. ${ }^{66-68}$ Dr. Catherine Lozupone from the University of Colorado opened this session with a presentation about metabolic syndromes associated with HIV, the involvement of microbiome components and the possibility of dietary interventions. Using a cohort of HIV+ and HIV- 
men who have sex with men (MSM) and men who have sex with women (MSW), she generated a metabolic score from each individual's metabolic parameters and compared it to their microbiome, diet, clinical data, and cytokine profiles. Lipopolysaccharide (LPS)-binding protein was found to be an important driver of metabolic score and also correlated positively with Dorea and Oscillospira and negatively with butyrate producers Coprococcus cactus and Butyrivibrio. Metabolome analysis of humanized mice fed with a high fat diet revealed further linkage between inflammatory cytokines, Dorea species and metabolic score. Diet intervention studies are ongoing in MSM and MSW living with HIV in hopes of creating personalized approaches to metabolic health in these populations at high risk of metabolic disorders.

Dr. Jean-Pierre Routy from McGill University spoke about metabolic endotoxemia in individuals living with HIV focusing on the little known topic of fungal translocation. Circulating (1-3) $\beta$-D-glucan (BDG) was found to be associated with immune activation in HIV infection. ${ }^{69}$ Other studies have also found associations between BDG and cardiopulmonary and neurocognitive function in PLHIV. ${ }^{70,71}$ BDG was also positively associated with marker for bacterial translocation (LPS), gut integrity (IFABP, IDO activity), and immune activation (CD8, Treg). ${ }^{69}$ BDGs are constituents of many foods such as oats, dates, and bananas. However, oral intake did not modify BDG plasma levels. Increased microbial translocation and increased levels of LPS and BDG were also associated with cytomegalovirus seropositivity. ${ }^{72}$ Thus future therapeutic strategies may include dietary interventions, probiotics, and blockage of cytomegalovirus replication.

Other notable data presented in poster format included reports of the partial reversal of gut microbial dysbiosis in rhesus macaques following antiretroviral therapy (Dr. Joseph Mattapallil from the Uniformed Services University); lower alpha-diversity in the rectal microbiome of MSM living with HIV in Kenya irrespective of antiretroviral treatment (Henok Gebrebrhan from the University of Manitoba); the effects of SIV infection and antiretroviral treatment on rhesus macaque gut microbiota (Dr. Sammer Siddiqui from the Tulane National Primate Research Center); the effects of vedolizumab on the gut microbiome (Dr. Marissa Becker from the University of Manitoba); a Streptococcus dominant dysbiotic gut microbiome in a population of antiretroviral experienced individuals from Ghana (Prince Parbie from Kumamoto University); the bioinformatic packages Nephele and METAGENOME for microbiome data analysis and deposition (Dr. Mariam Quiñones from NIAID/NIH) and associations of gut microbial communities with CD4 counts in children living with HIV in Cameroon (Baiye William Abange from the University of Buea).

Microbial dysbiosis and related morbidities are common in HIV+ individuals, ${ }^{73,74}$ however, sexual practices have also been linked to microbiome alterations HIV. ${ }^{75-77}$ To begin to distinguish sequelae of HIV from other causes, Jennifer Schneider from the University of Colorado reported that both HIV- and HIV+ MSM self-report gastrointestinal symptoms at a higher rate and have higher average fecal calprotectin levels than HIV-MSW in her cohort. Further, a wide assay of cytokines, chemokines, growth factors, and other potential biomarkers in fecal samples found that MSM clustered separately of MSW independently of HIV status.

Dr. Ivan Vujkovic-Cvijin from the NIAID/NIH also worked to distinguish changes in the gut microbiome due to HIV infection from those due to sex or sexual practices. In a cohort of PLHIV and uninfected participants, HIV+ individuals clustered separately from HIV- even when broken out by MSM, MSW, and females. Microbiota shifts in MSM appeared distinct from microbial shifts associated with HIV infection. The microbial communities also showed signs of further shifts between MSM with and without recent receptive anal intercourse. In addition, the microbiota of females engaging in recent receptive anal intercourse shared similarities with that of MSM. Thus, sexual practice appears to be associated with specific microbiome shifts that are distinct from those associated with HIV infection.

Preliminary data presented in poster format in this category included a study describing an association of plasma lipidomic profiles with markers of disease progression and inflammation in SIV-infected pig-tailed macaques (Dr. Ivona Vasile-Pandrea from the University of Pittsburgh) and a study of intestinal parasitic infections among children living with HIV in Ethiopia (Dr. Tsegaye Alemayehu from Hawassa University).

\section{Therapeutics}

Microbial dysbioses in PLHIV have been linked to the translocation of microbes from the gut and chronic inflammation. ${ }^{57-59}$ Therefore, modulation of the microbiome offers a potential therapeutic approach. However, published data to date are contradictory as to whether a "healthy" microbiome can be stably reconstituted and whether it can reduce systemic inflammation, ${ }^{78-81}$ so further trials are essential.

Dr. Stephane Isnard from McGill University discussed the possibility of using metformin treatment as an antiinflammatory agent to shift gut microbiota. Metformin is a first-line medication for the treatment of type 2 diabetes mellitus. ${ }^{82}$ It appears to modulate the gut microbiome ${ }^{83,84}$ and has shown anti-inflammatory and antiaging effects in animal models. ${ }^{85,86}$ In an open label, nonrandomized, single-arm clinical trial (Lilac study), 22 virologically suppressed individuals were treated with metformin for 12 weeks. ${ }^{87}$ Metformin was well tolerated, and there were no significant changes in CD4 or CD8 counts, glycohemoglobin, or fasting glucose levels. Analysis of stool samples demonstrated potential increases in bacterial diversity and slight changes in fungal microbiota following treatment. This pilot study will inform the design of studies with longer treatment durations.

Another potential approach is the fecal microbiota transplant (FMT), which has been used to successfully treat several diseases, including ulcerative colitis and recurrent Clostridium difficile infection. ${ }^{88-92}$ Dr. Ma Somsouk from University of California, San Francisco, discussed a clinical trial conducted to test the effectiveness of FMT in reducing microbial dysbiosis. Six virologically suppressed HIV+ individuals with low CD4 counts were transplanted with fecal microbiota from donors with low Proteobacteria and low Prevotella abundance (NCT02256592). ${ }^{93}$ Although the communities of the recipients shifted to be slightly closer to 
those of the donor, the changes were relatively minor compared to the initial differences between donors and recipients. In comparison, FMT into $C$. difficile patients displayed much larger shifts of gut communities toward those of fecal donors. Since $C$. difficile patients display much less pretreatment diversity and are antibiotic-treated, the study was repeated with a pretreatment of antibiotics. Again little to no community engraftment was observed. Based on previous studies in HIV-uninfected individuals with larger degrees of engraftment, future studies may wish to consider the use of "superdonors,"94,95 anaerobic stool processing, 92 pooled donors, ${ }^{92}$ repeat dosing, ${ }^{90,92,94}$ or probiotics. ${ }^{96-99}$

Dr. Sergio Serrano-Villar from Hospital Universitario Ramón y Cajal also described an ongoing phase I/II randomized, double-blind, placebo-controlled study of FMT in HIV+ individuals (REFRESH, REpeated low-dose Fecal microbiota REStoration in Hiv; NCT0300894). Fecal donors were selected from OpenBiomes's Universal Stool Bank based on high abundance of Faecalibacterium prausnitzii, Bacteroides species and butyrate producers with a low abundance of Prevotella species. Oral capsules were administered weekly for 7 weeks to 47 individuals living with HIV well suppressed by antiretroviral treatment. The product was well-tolerated. At the end of 48 weeks, there were modest signs of engraftment, but little change was observed in CD4/CD8 profiles and plasma inflammatory biomarkers. Engraftment may have been facilitated by recent antibiotic use in some patients. Future steps include larger studies with higher doses for longer period, careful evaluation of donors, and recommendations for antibiotic preconditioning.

Dr. Netenya Utay from University of Texas Health Science Center at Houston described another FMT approach using PRIM-DJ2727, an oral encapsulated lyophilized fecal microbiota product derived from healthy human donors with a high efficacy rate in refractory $C$. difficile infection. Six weekly doses in six virologically suppressed, HIV+ MSM individuals were well tolerated. Modest but transitory changes in microbiota were observed and biomarkers of inflammation and gut damage did not change significantly. Further studies are required to optimize donor and recipient characteristics as well as dosage, antibiotic pretreatment, and prebiotic dietary supplementation.

Although FMT provide an effective treatment for several diseases, the use of fecal material brings difficulties in standardization, safety testing, and administration. This is highlighted by the recent transfer of drug-resistant Escherichia coli into two FMT recipients and subsequent death of one of the recipients ${ }^{100}$ and a previous FMTrelated death due to aspiration of fecal material. ${ }^{101}$ Several pharmaceutical companies are working to develop defined communities of bacterial strains to provide a standardized alternative to FMT. For example, Dr. Scott Plevy provided an insider's view of the work at Janssen and Synlogic to bring live biotherapeutic products (LBP) into the clinic. Dr. Plevy described the work required to bring a LBP consisting of 11 or 16 Clostridial strains ${ }^{102-104}$ called VE202 to market. From acquisition of the strains in 2015, the Janssen team was able to bring the LBP to a first-in-human trial by 2018. As another example, Dr. Plevy described the work at Synlogic to develop genetically modified strains of $E$. coli Nissle $^{105}$ suitable for use in humans. After modifying the bacteria to be dependent on exogenous amino acids, the team has engineered a strain capable of breaking down phenylalanine that has shown promise in the treatment of patients with phenyl ketone urea. ${ }^{106}$

\section{Vaccines}

The targeted development of broadly neutralizing antibodies to HIV-1 remains one of the great challenges in the field. ${ }^{107-110}$ Dr. Wilton Williams, from Duke University, discussed mechanisms by which B cell response to vaccine antigens may be misled by cross-reactivity with intestinal microbiota. In acute infection, the initial ineffective antibody response to HIV largely develops from B cells polyreactive to both intestinal microbiota and HIV Env gp41. ${ }^{97,111-113}$ Similar polyreactive antibodies develop after immunization with a DNA prime-recombinant adenovirus type 5 boost (DNA/rAd5) vaccination, ${ }^{114}$ perhaps helping to explain the vaccine's lack of efficacy. ${ }^{115}$ Polyreactive gp41 antibodies also develop in a macaque model, even in neonatal macaques vaccinated shortly after birth concurrent with initial gut colonization. ${ }^{116}$ Thus, future vaccine designs may need to avoid antigens cross-reactive with host microbiome.

Recent studies have indicated that microbiome perturbations can increase inflammation and negatively impact vaccine responsiveness. ${ }^{117-120}$ Mouse studies in Dr. Daria Hazuda's laboratory at Merck demonstrated differential effects of classes of antibiotics on response to OVA antigen and hepatitis B vaccine, with the most significant hyporesponsiveness observed in vancomycin-treated mice. Vancomycintreated rhesus macaques also demonstrated reduced flu vaccine outcomes. However, IgG titers recovered when a flu booster vaccine was administered in the absence of vancomycin. Poor vaccine response was also observed in germ-free mice, however, titers to the vaccine returned to normal levels after fecal microbial transplant from untreated mice but not from mice treated with vancomycin. Finally, just as aging humans have reduced vaccine responses, ${ }^{121}$ an aging mouse model demonstrated hyporesponsiveness to OVA, flu vaccine, and HepA/B vaccine.

\section{Discussion}

The fifth year of this meeting ended with a discussion regarding "what have we learned in the last five years and what is the way forward?" The field was excited to see live therapeutic products, such as Lactin V, move forward in clinical trials. There was agreement regarding the importance of developing the concept of microbiome to include not only the commonly studied bacteriome but also the virome and the mycobiome. The probiotics studies have produced mixed messages so far and there is an overall need for consistent study design as well as consistent and standardized definitions and nomenclature. Choosing the appropriate in vitro, ex vivo, and in vivo models are critical, especially when interpolating the findings for humans. For example, microbiome in mice and rats are so different that the findings in such models may not translate well into humans. Important clarification has come from the recognition that behavioral factors may impact the microbiome and obscure the effects of HIV status. While a role for the vaginal microbiome in 
transmission seems likely, cause versus consequence in the relationship between gut dysbiosis and HIV pathogenesis still needs to be established. Scientific guidance on which products should move into clinical trials is currently lacking. Also, lacking is consistent incorporation of critical variables such as race, ethnicity, gender, and age when designing studies. As the population living with HIV is aging, ageassociated comorbidities and risk factors become particularly important. To begin addressing these concerns, greater involvement of community members in research should be encouraged, particularly increased participation at the stage of study design.

\section{Perspectives from the Community}

The conference was strengthened by the participation of community representative David Crawford whose role as Treatments Officer at Positive Life NSW, is to explore and inform the members of his community in Australia regarding recent research on current and emerging health issues that translate into education and service development for PLHIV. Mr. Crawford has been attending this conference since 2015 and noted that his community always appreciates the information he brings back, long before they hear about it from their physicians. From his community perspective, Mr. Crawford was disheartened by the lack of progress in studies regarding the role of diet on the microbiome (noting that the 2017 workshop presented data from animal studies on high fat diets, and the impact of neurological and mental health HIV in relationship to the gut-brain axis). This reflects a gap between scientific research agendas and expectations of community. Mr. Crawford reports that many members of his community took information he presented from earlier workshops and modified their own diets, such as using cruciferous vegetables for potential mental health improvement, lowering red meat intake, and other ways of trying to address the impacts of ongoing inflammation. These initiatives can be vastly improved if supported by translational research. Therefore, from a community perspective, scientific studies that will translate into recommended dietary guidelines for PLHIV is a critical gap. Another important direction of research may be behavior. While some presentations explored the microbiome and behavioral correlates, potential confounders were overlooked in these studies that detracted from the findings. It would be advantageous to have PLHIV more involved in all stages of research from design through to publication. This would, in part, address misaligned expectations and lead to better informed research design when exploring the relationship between behavior and impacts on the "biomes", which would lead to a richer outcome in findings. Finally, acknowledging that research participants are important and create an inclusive atmosphere and drive collaboration between the researcher and the community. If the intended research does not meet the expectations of both sides, there will be an imbalance in the outcome.

\section{Conclusion}

Overall, the broad range of topics discussed in this meeting shows that research detailing the interplay between the mi- crobiome and HIV infection is driving diverse approaches to therapeutically modulate the microbiome and potentially decrease transmission and comorbidities. HIV microbiome research has rapidly expanded from observational studies into treatments in various stages of human trials. Further discoveries of the roles of microbiome in HIV disease and the development of better models, diagnostics, and treatments will require continuing collaboration from a broad coalition of researchers with expertise spanning many fields in cooperation with the diverse communities of people living with or at risk from HIV.

\section{Author Disclosure Statement}

No competing financial interests exist.

\section{Funding Information}

Virology Education provided a travel grant to the first author to attend the conference.

\section{References}

1. Williams B, Mirmonsef $\mathrm{P}$, Boucher $\mathrm{CAB}$, et al.: A summary of the first HIV Microbiome workshop 2015. AIDS Res Hum Retroviruses 2016;32:935-941.

2. Williams B, Ghosh M, Boucher CAB, et al.: A summary of the second annual HIV Microbiome workshop. AIDS Res Hum Retroviruses 2017;33:1258-1264.

3. Williams B, Boucher C, Bushman F, et al.: A summary of the third annual HIV Microbiome workshop. AIDS Res Hum Retroviruses 2018;34:828-834.

4. Williams B, Ghosh M, Boucher CAB, et al.: A summary of the fourth annual Virology Education HIV Microbiome workshop. AIDS Res Hum Retroviruses 2020;36:349356.

5. Taha TE, Hoover DR, Dallabetta GA, et al.: Bacterial vaginosis and disturbances of vaginal flora: Association with increased acquisition of HIV. AIDS 1998;12:16991706.

6. Martin HL, Richardson BA, Nyange PM, et al.: Vaginal lactobacilli, microbial flora, and risk of human immunodeficiency virus type 1 and sexually transmitted disease acquisition. J Infect Dis 1999;180:1863-1868.

7. Atashili J, Poole C, Ndumbe PM, Adimora AA, Smith JS: Bacterial vaginosis and HIV acquisition: A meta-analysis of published studies. AIDS 2008;22:1493-1501.

8. Gosmann C, Anahtar MN, Handley SA, et al.: Lactobacillus-deficient cervicovaginal bacterial communities are associated with increased HIV acquisition in young South African women. Immunity 2017;46: 29-37.

9. Klatt NR, Cheu R, Birse K, et al.: Vaginal bacteria modify HIV tenofovir microbicide efficacy in African women. Science 2017;356:938-945.

10. Carlson RD, Sheth AN, Read TD, et al.: The female genital tract microbiome is associated with vaginal antiretroviral drug concentrations in human immunodeficiency virus-infected women on antiretroviral therapy. J Infect Dis 2017;216:990-999.

11. Ravel J, Gajer P, Abdo Z, et al.: Vaginal microbiome of reproductive-age women. Proc Natl Acad Sci U S A 2011; 108(Suppl 1):4680-4687. 
12. Gajer P, Brotman RM, Bai G, et al.: Temporal dynamics of the human vaginal microbiota. Sci Transl Med 2012;4: 132 ra52.

13. Lennard K, Dabee S, Barnabas SL, et al: Microbial composition predicts genital tract inflammation and persistent bacterial vaginosis in South African adolescent females. Infect Immun 2018;86:e0410-17. DOI: 10.1128/IAI.00410-17.

14. Ma B, France M, Crabtree J, et al.: VIRGO, a comprehensive non-redundant gene catalog, reveals extensive within community intraspecies diversity in the human vagina. bioRxiv 2019;660498.

15. Li F, Chen $\mathrm{C}$, Wei $\mathrm{W}$, et al.: The metagenome of the female upper reproductive tract. GigaScience 2018;7: giy 107. DOI: 10.1093/gigascience/giy 107.

16. Brotman RM, Klebanoff MA, Nansel TR, et al:: A longitudinal study of vaginal douching and bacterial vaginosis-A marginal structural modeling analysis. Am J Epidemiol 2008;168:188-196.

17. Potter RF, Burnham CAD, Dantas G: In silico analysis of Gardnerella genomospecies detected in the setting of bacterial vaginosis. Clin Chem 2019;65:1375-1387.

18. Gong Z, Luna Y, Yu P, Fan H: Lactobacilli inactivate Chlamydia trachomatis through lactic acid but not $\mathrm{H}_{2} \mathrm{O}_{2}$. PLoS One 2014;9:e107758.

19. Mastromarino P, Di Pietro M, Schiavoni G, Nardis C, Gentile M, Sessa R: Effects of vaginal lactobacilli in Chlamydia trachomatis infection. Int $\mathrm{J}$ Med Microbiol 2014;304:654-661.

20. Edwards VL, Smith SB, McComb EJ, et al.: The cervicovaginal microbiota-host interaction modulates Chlamydia trachomatis infection. mBio 2019;10:e0154819. DOI: $10.1128 / \mathrm{mBio} .01548-19$.

21. Anahtar MN, Byrne EH, Doherty KE, et al.: Cervicovaginal bacteria are a major modulator of host inflammatory responses in the female genital tract. Immunity 2015;42:965-976.

22. Masson L, Passmore JAS, Liebenberg LJ, et al.: Genital inflammation and the risk of HIV acquisition in women. Clin Infect Dis 2015;61:260-269.

23. Bukusi E, Thomas KK, Nguti R, et al.: Topical penile microbicide use by men to prevent recurrent bacterial vaginosis in sex partners: A randomized clinical trial. Sex Transm Dis 2011;38:483-489.

24. McClelland RS, Balkus JE, Lee J, et al.: Randomized trial of periodic presumptive treatment with high-dose intravaginal metronidazole and miconazole to prevent vaginal infections in HIV-negative women. J Infect Dis 2015; 211:1875-1882.

25. Francis SC, Looker C, Vandepitte $\mathrm{J}$, et al.: Bacterial vaginosis among women at high risk for HIV in Uganda: High rate of recurrent diagnosis despite treatment. Sex Transm Infect 2016;92:142-148.

26. Hemmerling A, Harrison W, Schroeder A, et al.: Phase 1 dose-ranging safety trial of Lactobacillus crispatus CTV05 for the prevention of bacterial vaginosis. Sex Transm Dis 2009;36:564-569.

27. Hemmerling A, Harrison W, Schroeder A, et al.: Phase 2a study assessing colonization efficiency, safety, and acceptability of Lactobacillus crispatus CTV-05 in women with bacterial vaginosis. Sex Transm Dis 2010;37:745750 .

28. Ngugi BM, Hemmerling A, Bukusi EA, et al.: Effects of bacterial vaginosis-associated bacteria and sexual inter- course on vaginal colonization with the probiotic Lactobacillus crispatus CTV-05. Sex Transm Dis 2011;38: 1020-1027.

29. Cohen CR, Wierzbicki MR, French AL, et al.: Randomized trial of Lactin-V to prevent recurrence of bacterial vaginosis. N Engl J Med 2020;382:1906-1915.

30. Kenyon C, Colebunders R, Crucitti T: The global epidemiology of bacterial vaginosis: A systematic review. Am J Obstet Gynecol 2013;209:505-523.

31. Nel A, van Niekerk N, Kapiga S, et al.: Safety and efficacy of a dapivirine vaginal ring for HIV prevention in women. N Engl J Med 2016;375:2133-2143.

32. Baeten JM, Palanee-Phillips T, Brown ER, et al.: Use of a vaginal ring containing dapivirine for HIV-1 prevention in women. N Engl J Med 2016;375:2121-2132.

33. Baum MM, Butkyavichene I, Gilman J, et al.: An intravaginal ring for the simultaneous delivery of multiple drugs. J Pharm Sci 2012;101:2833-2843.

34. Srinivasan P, Moss JA, Gunawardana M, et al.: Topical delivery of tenofovir disoproxil fumarate and emtricitabine from pod-intravaginal rings protects macaques from multiple SHIV exposures. PLoS One 2016;11: e0157061.

35. Vincent KL, Moss JA, Marzinke MA, et al:: Safety and pharmacokinetics of single, dual, and triple antiretroviral drug formulations delivered by pod-intravaginal rings designed for HIV-1 prevention: A phase I trial. PLoS Med 2018;15:e1002655.

36. Delgado-Diaz DJ, Tyssen D, Hayward JA, Gugasyan R, Hearps AC, Tachedjian G: Distinct immune responses elicited from cervicovaginal epithelial cells by lactic acid and short chain fatty acids associated with optimal and non-optimal vaginal microbiota. Front Cell Infect Microbiol 2020;9:446.

37. Moutsopoulos NM, Konkel JE: Tissue-specific immunity at the oral mucosal barrier. Trends Immunol 2018;39:276287.

38. Silva LM, Brenchley L, Moutsopoulos NM: Primary immunodeficiencies reveal the essential role of tissue neutrophils in periodontitis. Immunol Rev 2019;287: 226-235.

39. Veldhoen M: Interleukin 17 is a chief orchestrator of immunity. Nat Immunol 2017;18:612-621.

40. Stockinger B, Omenetti S: The dichotomous nature of T helper 17 cells. Nat Rev Immunol 2017;17:535-544.

41. Dutzan N, Konkel JE, Greenwell-Wild T, Moutsopoulos NM: Characterization of the human immune cell network at the gingival barrier. Mucosal Immunol 2016;9:11631172.

42. Dutzan N, Abusleme L, Bridgeman H, et al:: On-going mechanical damage from mastication drives homeostatic Th17 cell responses at the oral barrier. Immunity 2017;46: 133-147.

43. Dutzan N, Kajikawa T, Abusleme L, et al.: A dysbiotic microbiome triggers Th17 cells to mediate oral mucosal immunopathology in mice and humans. Sci Transl Med 2018;10:eaat0797. DOI: 10.1126/scitranslmed.aat0797.

44. Milner JD, Brenchley JM, Laurence A, et al.: Impaired $\mathrm{T}(\mathrm{H}) 17$ cell differentiation in subjects with autosomal dominant hyper-IgE syndrome. Nature 2008;452:773776.

45. Belkaid Y, Hand TW: Role of the microbiota in immunity and inflammation. Cell 2014;157:121-141. 
46. Kamada N, Seo SU, Chen GY, Núñez G: Role of the gut microbiota in immunity and inflammatory disease. Nat Rev Immunol 2013;13:321-335.

47. Pfeiffer JK, Virgin HW: Transkingdom control of viral infection and immunity in the mammalian intestine. Science 2016;351:aad5872. DOI: 10.1126/science.aad5872.

48. Robinson CM: Enteric viruses exploit the microbiota to promote infection. Curr Opin Virol 2019;37:58-62.

49. Walker FC, Baldridge MT: Interactions between noroviruses, the host, and the microbiota. Curr Opin Virol 2019;37:1-9.

50. Lee S, Wilen CB, Orvedahl A, et al.: Norovirus cell tropism is determined by combinatorial action of a viral nonstructural protein and host cytokine. Cell Host Microbe 2017;22:449.e4-459.e4.

51. Orchard RC, Wilen CB, Doench JG, et al:: Discovery of a proteinaceous cellular receptor for a norovirus. Science 2016;353:933-936.

52. Wilen CB, Lee S, Hsieh LL, et al.: Tropism for tuft cells determines immune promotion of norovirus pathogenesis. Science 2018;360:204-208.

53. Lei W, Ren W, Ohmoto M, et al:: Activation of intestinal tuft cell-expressed Sucnr1 triggers type 2 immunity in the mouse small intestine. Proc Natl Acad Sci U S A 2018; 115:5552-5557.

54. Nadjsombati MS, McGinty JW, Lyons-Cohen MR, et al.: Detection of succinate by intestinal tuft cells triggers a type 2 innate immune circuit. Immunity 2018;49:33. e7-41.e7.

55. Schneider C, O'Leary CE, von Moltke J, et al.: A metabolite-triggered tuft cell-ILC2 circuit drives small intestinal remodeling. Cell 2018;174:271.e14-284.e14.

56. Ingle $\mathrm{H}$, Lee $\mathrm{S}, \mathrm{Ai} \mathrm{T}$, et al.: Viral complementation of immunodeficiency confers protection against enteric pathogens via interferon- $\lambda$. Nat Microbiol 2019;4:1120 1128.

57. Brenchley JM, Price DA, Schacker TW, et al.: Microbial translocation is a cause of systemic immune activation in chronic HIV infection. Nat Med 2006;12:1365-1371.

58. Estes JD, Harris LD, Klatt NR, et al.: Damaged intestinal epithelial integrity linked to microbial translocation in pathogenic simian immunodeficiency virus infections. PLoS Pathog 2010;6:e1001052.

59. Klase Z, Ortiz A, Deleage C, et al.: Dysbiotic bacteria translocate in progressive SIV infection. Mucosal Immunol 2015;8:1009-1020.

60. van Niel G, Raposo G, Candalh C, et al.: Intestinal epithelial cells secrete exosome-like vesicles. Gastroenterology 2001;121:337-349.

61. Mallegol J, Van Niel G, Lebreton C, et al.: T84-intestinal epithelial exosomes bear MHC class II/peptide complexes potentiating antigen presentation by dendritic cells. Gastroenterology 2007;132:1866-1876.

62. Hu G, Gong AY, Roth AL, et al.: Release of luminal exosomes contributes to TLR4-mediated epithelial antimicrobial defense. PLoS Pathog 2013;9:e1003261.

63. Smythies LE, Smythies JR: Exosomes in the gut. Front Immunol 2014;5:104.

64. Goto Y, Uematsu S, Kiyono H: Epithelial glycosylation in gut homeostasis and inflammation. Nat Immunol 2016;17: 1244-1251.

65. Giron LB, Tanes CE, Schleimann MH, et al.: Sialylation and fucosylation modulate inflammasome-activating eIF2 signaling and microbial translocation during HIV infection. Mucosal Immunol 2020;13:753-766.

66. Lai S, Bartlett J, Lai $\mathrm{H}$, et al:: Long-term combination antiretroviral therapy is associated with the risk of coronary plaques in African Americans with HIV infection. AIDS Patient Care STDS 2009;23:815-824.

67. Pao V, Lee GA, Grunfeld C: HIV therapy, metabolic syndrome, and cardiovascular risk. Curr Atheroscler Rep 2008;10:61-70.

68. Samaras K, Wand H, Law M, Emery S, Cooper D, Carr A: Prevalence of metabolic syndrome in HIV-infected patients receiving highly active antiretroviral therapy using International Diabetes Foundation and Adult Treatment Panel III criteria: Associations with insulin resistance, disturbed body fat compartmentalization, elevated C-reactive protein, and hypoadiponectinemia. Diabetes Care 2007;30:113-119.

69. Mehraj V, Ramendra R, Isnard S, et al.: Circulating $(1 \rightarrow 3)-\beta$-D-glucan is associated with immune activation during human immunodeficiency virus infection. Clin Infect Dis 2020;70:232-241.

70. Morris A, Hillenbrand M, Finkelman M, et al.: Serum $(1 \rightarrow 3)$ - $\beta$-D-glucan levels in HIV-infected individuals are associated with immunosuppression, inflammation, and cardiopulmonary function. J Acquir Immune Defic Syndr 2012;61:462-468.

71. Hoenigl M, de Oliveira MF, Pérez-Santiago J, et al.: $(1 \rightarrow 3)$ - $\beta$-D-glucan levels correlate with neurocognitive functioning in HIV-infected persons on suppressive antiretroviral therapy: A cohort study. Medicine 2016;95: e3162.

72. Ramendra R, Isnard S, Lin J, et al.: CMV seropositivity is associated with increased microbial translocation in people living with HIV and uninfected controls. Clin Infect Dis 2019;ciz1001. DOI: 10.1093/cid/ciz1001.

73. Mudd JC, Brenchley JM: Gut mucosal barrier dysfunction, microbial dysbiosis, and their role in HIV-1 disease progression. J Infect Dis 2016;214(Suppl 2): S58-S66.

74. Tincati C, Douek DC, Marchetti G: Gut barrier structure, mucosal immunity and intestinal microbiota in the pathogenesis and treatment of HIV infection. AIDS Res Ther 2016;13:19.

75. Noguera-Julian $\mathrm{M}$, Rocafort $\mathrm{M}$, Guillén $\mathrm{Y}$, et al.: Gut microbiota linked to sexual preference and HIV infection. EBioMedicine 2016;5:135-146.

76. Kelley CF, Kraft CS, de Man TJB, et al:: The rectal mucosa and condomless receptive anal intercourse in HIV-negative MSM: Implications for HIV transmission and prevention. Mucosal Immunol 2017;10:996-1007.

77. Armstrong AJS, Shaffer M, Nusbacher NM, et al:: An exploration of Prevotella-rich microbiomes in HIV and men who have sex with men. Microbiome 2018; 6:198.

78. Tenorio AR, Chan ES, Bosch RJ, et al:: Rifaximin has a marginal impact on microbial translocation, T-cell activation and inflammation in HIV-positive immune nonresponders to antiretroviral therapy-ACTG A5286. J Infect Dis 2015;211:780-790.

79. Hensley-McBain T, Zevin AS, Manuzak J, et al.: Effects of fecal microbial transplantation on microbiome and immunity in simian immunodeficiency virus-infected macaques. J Virol 2016;90:4981-4989. 
80. Ortiz AM, Flynn JK, DiNapoli SR, et al.: Experimental microbial dysbiosis does not promote disease progression in SIV-infected macaques. Nat Med 2018;24:13131316.

81. Bourke CD, Gough EK, Pimundu G, et al.: Cotrimoxazole reduces systemic inflammation in HIV infection by altering the gut microbiome and immune activation. Sci Transl Med 2019;11:eaav0537. DOI: 10.1126/scitranslmed.aav0537.

82. Maruthur NM, Tseng E, Hutfless S, et al:: Diabetes medications as monotherapy or metformin-based combination therapy for type 2 diabetes: A systematic review and meta-analysis. Ann Intern Med 2016;164: 740-751.

83. Forslund K, Hildebrand F, Nielsen T, et al.: Disentangling type 2 diabetes and metformin treatment signatures in the human gut microbiota. Nature 2015;528:262-266.

84. Cabreiro F: Metformin joins forces with microbes. Cell Host Microbe 2016;19:1-3.

85. De Haes W, Frooninckx L, Van Assche R, et al:: Metformin promotes lifespan through mitohormesis via the peroxiredoxin PRDX-2. Proc Natl Acad Sci U S A 2014; 111:E2501-E2509.

86. Moyo D, Tanthuma G, Cary MS, et al.: Cohort study of diabetes in HIV-infected adult patients: Evaluating the effect of diabetes mellitus on immune reconstitution. Diabetes Res Clin Pract 2014;103:e34-e36.

87. Routy J-P, Isnard S, Mehraj V, et al.: Effect of metformin on the size of the HIV reservoir in non-diabetic ARTtreated individuals: Single-arm non-randomised Lilac pilot study protocol. BMJ Open 2019;9:e028444.

88. DeFilipp Z, Peled JU, Li S, et al.: Third-party fecal microbiota transplantation following allo-HCT reconstitutes microbiome diversity. Blood Adv 2018;2:745753.

89. van Nood E, Vrieze A, Nieuwdorp M, et al.: Duodenal infusion of donor feces for recurrent Clostridium difficile. N Engl J Med 2013;368:407-415.

90. Moayyedi P, Surette MG, Kim PT, et al.: Fecal microbiota transplantation induces remission in patients with active ulcerative colitis in a randomized controlled trial. Gastroenterology 2015;149:102.e6-109.e6.

91. Paramsothy S, Kamm MA, Kaakoush NO, et al.: Multidonor intensive faecal microbiota transplantation for active ulcerative colitis: A randomised placebo-controlled trial. Lancet 2017;389:1218-1228.

92. Costello SP, Hughes PA, Waters O, et al.: Effect of fecal microbiota transplantation on 8 -week remission in patients with ulcerative colitis: A randomized clinical trial. JAMA 2019;321:156-164.

93. Vujkovic-Cvijin I, Rutishauser RL, Pao M, et al.: Limited engraftment of donor microbiome via one-time fecal microbial transplantation in treated HIV-infected individuals. Gut Microbes 2017;8:440-450.

94. Rossen NG, Fuentes S, van der Spek MJ, et al.: Findings from a randomized controlled trial of fecal transplantation for patients with ulcerative colitis. Gastroenterology 2015; 149:110.e4-118.e4.

95. Wilson BC, Vatanen T, Cutfield WS, O'Sullivan JM: The super-donor phenomenon in fecal microbiota transplantation. Front Cell Infect Microbiol 2019;9:2.

96. Panigrahi P, Parida S, Nanda NC, et al.: Role of probiotics VSL\#3 in prevention of suspected sepsis in low birth- weight infants in India: A randomised controlled trial. Nature 2017;548:407-412.

97. Jacobs SE, Tobin JM, Opie GF, et al:: Probiotic effects on late-onset sepsis in very preterm infants: A randomized controlled trial. Pediatrics 2013;132:1055-1062.

98. Costeloe K, Hardy P, Juszczak E, Wilks M, Millar MR, Probiotics in Preterm Infants Study Collaborative Group: Bifidobacterium breve BBG-001 in very preterm infants: A randomised controlled phase 3 trial. Lancet 2016;387: 649-660.

99. Sinha A, Gupta Subodh S, Chellani H, et al.: Role of probiotics VSL\#3 in prevention of suspected sepsis in low birthweight infants in India: A randomised controlled trial. BMJ Open 2015;5:e06564.

100. DeFilipp Z, Bloom PP, Soto MT, et al.: Drug-resistant E. coli bacteremia transmitted by fecal microbiota transplant. N Engl J Med 2019;381:2043-2050.

101. Baxter M, Ahmad T, Colville A, Sheridan R: Fatal aspiration pneumonia as a complication of fecal microbiota transplant. Clin Infect Dis 2015;61:136-137.

102. Atarashi $\mathrm{K}$, Tanoue $\mathrm{T}$, Shima $\mathrm{T}$, et al: Induction of colonic regulatory $\mathrm{T}$ cells by indigenous Clostridium species. Science 2011;331:337-341.

103. Atarashi K, Tanoue T, Oshima K, et al:: Treg induction by a rationally selected mixture of Clostridia strains from the human microbiota. Nature 2013;500:232-236.

104. Ratner M: Microbial cocktails join fecal transplants in IBD treatment trials. Nat Biotechnol 2015;33:787788.

105. Kruis W, Fric P, Pokrotnieks J, et al.: Maintaining remission of ulcerative colitis with the probiotic Escherichia coli Nissle 1917 is as effective as with standard mesalazine. Gut 2004;53:1617-1623.

106. Bermúdez-Humarán Luis G, Langella P: Live bacterial biotherapeutics in the clinic. Nat Biotechnol 2018;36:816818.

107. Mascola JR, Haynes BF: HIV-1 neutralizing antibodies: Understanding nature's pathways. Immunol Rev 2013; 254:225-244.

108. Havenar-Daughton C, Lee JH, Crotty S: Tfh cells and HIV bnAbs, an immunodominance model of the HIV neutralizing antibody generation problem. Immunol Rev 2017; 275:49-61.

109. Burton DR, Hangartner L: Broadly neutralizing antibodies to HIV and their role in vaccine design. Annu Rev Immunol 2016;34:635-659.

110. Haynes BF, Burton DR: Developing an HIV vaccine. Science 2017;355:1129-1130.

111. Tomaras GD, Yates NL, Liu P, et al.: Initial B-cell responses to transmitted human immunodeficiency virus type 1: Virion-binding immunoglobulin $\mathrm{M}(\operatorname{IgM})$ and $\mathrm{IgG}$ antibodies followed by plasma anti-gp41 antibodies with ineffective control of initial viremia. J Virol 2008;82: 12449-12463.

112. Liao $\mathrm{H}-\mathrm{X}$, Chen $\mathrm{X}$, Munshaw $\mathrm{S}$, et al:: Initial antibodies binding to HIV-1 gp41 in acutely infected subjects are polyreactive and highly mutated. J Exp Med 2011;208: 2237-2249.

113. Trama AM, Moody MA, Alam SM, et al.: HIV-1 envelope gp41 antibodies can originate from terminal ileum B cells that share cross-reactivity with commensal bacteria. Cell Host Microbe 2014;16:215-226. 
114. Williams WB, Liao H-X, Moody MA, et al.: Diversion of HIV-1 vaccine-induced immunity by gp41-microbiota cross-reactive antibodies. Science 2015;349:aab1253.

115. Hammer SM, Sobieszczyk ME, Janes H, et al.: Efficacy trial of a DNA/rAd5 HIV-1 preventive vaccine. N Engl J Med 2013;369:2083-2092.

116. Han Q, Williams WB, Saunders KO, et al.: HIV DNAadenovirus multiclade envelope vaccine induces gp41 antibody immunodominance in rhesus macaques. J Virol 2017;91:e00923-17. DOI: 10.1128/JVI.00923-17.

117. Hagan $\mathrm{T}$, Cortese M, Rouphael N, et al.: Antibioticsdriven gut microbiome perturbation alters immunity to vaccines in humans. Cell 2019;178:1313.e13-1328.e13.

118. Thackray LB, Handley SA, Gorman MJ, et al.: Oral antibiotic treatment of mice exacerbates the disease severity of multiple flavivirus infections. Cell Rep 2018;22: 3440.e6-3453.e6.

119. Lynn MA, Tumes DJ, Choo JM, et al:: Early-life antibiotic-driven dysbiosis leads to dysregulated vaccine immune responses in mice. Cell Host Microbe 2018;23: 653.e5-660.e5.
120. Yang JH, Bhargava P, McCloskey D, Mao N, Palsson BO, Collins JJ: Antibiotic-induced changes to the host metabolic environment inhibit drug efficacy and alter immune function. Cell Host Microbe 2017;22:757.e3765.e3.

121. Siegrist CA, Aspinall R: B-cell responses to vaccination at the extremes of age. Nat Rev Immunol 2009;9:185194.

Address correspondence to: Scott Sherrill-Mix

Department of Microbiology

Perelman School of Medicine

University of Pennsylvania 424 Johnson Pavilion 3610 Hamilton Walk

Philadelphia, Pennsylvania 19104

USA

E-mail: shescott@upenn.edu 